\title{
Anti-synchronization of fractional-order chaotic complex systems with unknown parameters via adaptive control
}

\author{
Cuimei Jiang ${ }^{a, b}$, Fangfang Zhang ${ }^{c}$, Haiyong Qin ${ }^{d}$, Tongxing Li $i^{e, f, *}$ \\ a School of Science, Qilu University of Technology, Jinan, Shandong 250353, P. R. China. \\ ${ }^{b}$ College of Mathematics and Systems Science, Shandong University of Science and Technology, Qingdao, Shandong 266590, P. R. \\ China. \\ ${ }^{c}$ School of Electrical Engineering and Automation, Qilu University of Technology, Jinan, Shandong 250353, P. R. China. \\ ${ }^{d}$ School of Mathematics, Qilu Normal University, Jinan, Shandong 250013, P. R. China. \\ ${ }^{e}$ LinDa Institute of Shandong Provincial Key Laboratory of Network Based Intelligent Computing, Linyi University, Linyi, Shandong \\ 276005, P. R. China. \\ ${ }^{f}$ School of Information Science and Engineering, Linyi University, Linyi, Shandong 276005, P. R. China.
}

Communicated by D. Baleanu

\begin{abstract}
This paper is concerned with adaptive control for anti-synchronization of a class of uncertain fractional-order chaotic complex systems described by a unified mathematical expression. By utilizing the recently established result for the Caputo fractional derivative of a quadratic function and employing the adaptive control technique, we design controllers and some fractional-order parameter update laws to anti-synchronize two fractional-order chaotic complex systems with unknown parameters. The proposed method has generality, simplicity, and feasibility. Moreover, anti-synchronization between uncertain fractional-order complex Lorenz system and fractional-order complex Lü system is implemented as an example to demonstrate the effectiveness and feasibility of the proposed scheme. (C)2017 All rights reserved.
\end{abstract}

Keywords: Adaptive control, anti-synchronization, fractional-order chaotic complex system, quadratic Lyapunov function. 2010 MSC: 34C28, 34D06.

\section{Introduction}

Based on the wide applications in the fields of secure communication, encryption, signal and control processing, fractional-order chaotic systems have attracted tremendous attention of scientists and engineers during the past few decades $[15,27,33]$. With the help of fractional-order calculus, it is demonstrated that there are many fractional-order chaotic systems including the fractional-order Chen system [17], the fractional-order financial system with time delay [34], the fractional-order hyperchaotic Rössler system [16], and so on. Meanwhile, synchronization has been a central issue in the study of fractionalorder chaotic systems and then has been extensively investigated. In previous works there are several

\footnotetext{
*Corresponding author

Email addresses: jiangcuimei2004@163.com (Cuimei Jiang), zhff4u@163.com (Fangfang Zhang), qhymath@163.com (Haiyong Qin), litongx2007@163.com (Tongxing Li)

doi:10.22436/jnsa.010.11.02
}

Received 2017-06-09 
different types of synchronization such as complete synchronization [11, 12, 35, 37], phase synchronization [32], impulsive synchronization [18], lag projective synchronization [6], just to enumerate a few examples. Among all kinds of chaos synchronization, anti-synchronization is one of the particular type of synchronization which was observed in periodic chaotic systems [42]. In anti-synchronization scheme, the state variables of synchronized systems with different initial values have the same absolute values but opposite signs. As a result, when anti-synchronization occurs, the sums of two signals are expected to converge to zero.

In contrast to the large amount of results in the literature on the fractional-order chaotic real systems, there are very few papers dealing with fractional-order systems with complex variables. Complex variables can be widely applied to describe a variety of physical phenomena; see, for instance, the atomic polarization amplitudes, electric field, population inversion, detuned laser systems, amplitudes of electromagnetic fields, thermal convections of liquid flows, etc. Therefore, many authors have taken complex variables into the fractional-order chaotic systems and concentrated on dynamics, chaos control, and synchronization of fractional-order complex nonlinear systems in recent years. The result shown in [10] dealt with chaotic dynamics of fractional order periodically forced complex Duffing's oscillators. It was known in [9] that dynamic properties of the fractional-order logistic equation of complex variables was analyzed. Luo and Wang [26] introduced the fractional-order complex Lorenz system and realized complete synchronization. The fractional-order complex Chen system was proposed and hybrid synchronization was applied to digital secure communication [25]. Liu et al. [20] obtained results on bifurcations, chaos control, and synchronization of the fractional-order complex $\mathrm{T}$ system. With respect to other related works on this topic, we refer the reader to $[13,14,19,21,31,40]$ and the references cited therein.

All of the above-mentioned papers concentrate on fractional-order chaotic complex systems with known parameters in priori, not involving unknown parameters. In fact, parameters of many systems cannot be exactly determined in advance in various engineering applications. And then the uncertainty of unknown parameters can destroy chaos synchronization. So unknown parameters have significantly negative effects on chaos communication and synchronization, for instance, in secure communication, the receiver will definitely suffer from uncertain parameters, which will no doubt influence the accuracy of the communication. Therefore, it is very important and challenging to overcome the uncertainty of unknown parameters to realize synchronization of the fractional-order chaotic complex systems. As far as we know, the adaptive control theory provides us with a useful and powerful tool to achieve synchronization of dynamical systems with unknown parameters. Up to now, many researchers have been concerned with adaptive synchronization of the fractional-order chaotic real systems, such as $[1,28,41]$ to name a few. However, to the best of our knowledge, there are few results about anti-synchronization of different fractional-order chaotic complex systems with unknown parameters.

Motivated by the above discussions, we investigate anti-synchronization between two different fractional-order chaotic complex systems with unknown parameters. It is necessary to point out that, unlike the schemes proposed in the previous literature [1, 28, 41], we construct appropriate quadratic Lyapunov functions to analyze the stability of fractional-order systems and utilize fractional-order adaptation laws to update design parameters.

The remainder of this paper is organized as follows. Section 2 reviews some preliminaries and gives the problem descriptions. In Section 3, we investigate adaptive anti-synchronization between two different fractional-order chaotic complex systems with all situations of unknown parameters. In Section 4, the proposed method is discussed numerically with the help of fractional-order chaotic complex Lorenz system and fractional-order chaotic complex Lü system with unknown parameters. Finally, conclusions are drawn in Section 5.

\section{Preliminaries}

\subsection{Fractional calculus and the stability of fractional-order systems}

As is known, the fractional derivative has three hundred years of history and fractional differential 
equations have been extensively studied due to their numerous applications in natural sciences and engineering [29]. Meanwhile, several definitions of fractional derivatives, such as the Grunwald-Letnikov definition, the Riemann-Liouville definition, and the Caputo definition, have been proposed. Amongst these definitions, the Caputo definition is used the most in engineering applications, since under this definition initial conditions have well-understood physical meaning. Hence, the Caputo derivative is chosen in this paper.

Definition 2.1 ([5]). The Caputo fractional derivative of order $\alpha \in \mathbb{R}^{+}$on the half axis $\mathbb{R}^{+}$is defined as follows:

$$
t_{0} D_{t}^{\alpha} f(t)=\frac{1}{\Gamma(n-\alpha)} \int_{t_{0}}^{t} \frac{f^{(n)}(\tau)}{(t-\tau)^{\alpha-n+1}} d \tau, \quad t>t_{0}
$$

where $n=\min \{k \in \mathbb{N} / k>\alpha\}, \Gamma$ stands for Gamma function, and $t_{0} D_{t}^{\alpha}$ is generally called $\alpha$-order Caputo differential operator.

With the rule of fractional-order calculus, the Caputo differential operator is a linear operator, which is described as follows:

$$
t_{0} D_{t}^{\alpha}(\lambda f(t)+\mu g(t))=\lambda_{t_{0}} D_{t}^{\alpha} f(t)+\mu_{t_{0}} D_{t}^{\alpha} g(t),
$$

where $\lambda$ and $\mu$ are real constants.

Recently, there have been many papers concerning on the stability of fractional-order systems $[2,3,7$, $22,23,36,39]$. Next, we give the subsequent lemmas which are helpful in proving our results.

Lemma 2.2 ([2]). Suppose that $x(t) \in \mathbb{R}$ is a continuous and derivable function. Then, for any time instant $\mathrm{t} \geqslant \mathrm{t}_{0}$,

$$
\frac{1}{2} \mathrm{t}_{0} \mathrm{D}_{\mathrm{t}}^{\alpha} \chi^{2}(\mathrm{t}) \leqslant \chi(\mathrm{t})_{\mathrm{t}_{0}} \mathrm{D}_{\mathrm{t}}^{\alpha} \chi(\mathrm{t}), \quad \forall \alpha \in(0,1) .
$$

Lemma 2.3 ([2]). Suppose that $x(t) \in \mathbb{R}^{n}$ is a continuous and derivable vector function. Then, for $\forall \alpha \in(0,1)$ and $\forall \mathrm{t} \geqslant \mathrm{t}_{0}$,

$$
\frac{1}{2} t_{0} D_{t}^{\alpha} x^{\top}(t) x(t) \leqslant x^{\top}(t)_{t_{0}} D_{t}^{\alpha} x(t)
$$

Lemma 2.4 ([24]). Let $\mathrm{W}(\mathrm{t})=\mathrm{x}^{\top}(\mathrm{t}) \mathrm{x}(\mathrm{t}) / 2+\mathrm{y}^{\top}(\mathrm{t}) \mathrm{y}(\mathrm{t}) / 2$, where $\mathrm{x}(\mathrm{t}), \mathrm{y}(\mathrm{t}) \in \mathbb{R}^{\mathrm{n}}$ have continuous derivatives. If there exists a constant $\mathrm{h}_{0}>0$ such that

$$
t_{0} D_{t}^{\alpha} W(t) \leqslant-h_{0} x^{\top}(t) x(t),
$$

then $\|x(t)\|$ and $\|\mathrm{y}(\mathrm{t})\|$ are bounded for all $\mathrm{t}>0$ and $\mathrm{x}(\mathrm{t})$ converges to zero asymptotically, where $\|\cdot\|$ is the Euclidean norm.

In this paper, we mainly consider the case of $0<\alpha<1$. For simplicity, we substitute $D_{*}^{\alpha}$ for $t_{0} D_{t}^{\alpha}$ and use the Adams-Bashforth-Moulton predictor-correctors scheme [8] to calculate the fractional differential equations.

\subsection{Mathematical model and problem descriptions}

Next, let us consider an $n$-dimensional fractional-order chaotic complex system with the controller as follows:

$$
D_{*}^{\alpha} y=G(y) B+g(y)+u,
$$

where $y=\left(y_{1}, y_{2}, \ldots, y_{n}\right)^{\top} \in \mathbb{C}^{n}$ is a complex state vector, $G(y) \in \mathbb{C}^{n \times n}$ is a complex matrix, $B=$ $\left(b_{1}, b_{2}, \ldots, b_{n}\right)^{\top}$ represents the real (or complex) vector of unknown parameters, and $g: \mathbb{C}^{n} \rightarrow \mathbb{C}^{n}$ describes the nonlinear term. $\mathrm{U}=\mathrm{U}^{\mathrm{r}}+j \mathrm{U}^{\mathrm{i}}$ is a complex controller to be designed, where $\mathrm{U}^{\mathrm{r}}=$ $\left(u_{1}, u_{3}, \ldots, u_{2 n-1}\right)^{\top}$ and $u^{i}=\left(u_{2}, u_{4}, \ldots, u_{2 n}\right)^{\top}$. Superscripts $r$ and $i$ stand for the real and imaginary parts of the complex state vector. System (2.1) is regarded as the response system and the drive system is described by

$$
\mathrm{D}_{*}^{\alpha} x=\mathrm{h}(x),
$$

where $x=\left(x_{1}, x_{2}, \ldots, x_{n}\right)^{\top} \in \mathbb{C}^{n}$ represents the complex state vector and $h: \mathbb{C}^{n} \rightarrow \mathbb{C}^{n}$ describes the nonlinear term. 
Remark 2.5. Most of the classical fractional-order chaotic complex systems can be written as the form of system (2.1), such as the fractional-order complex Lorenz system, the fractional-order complex Chen system, the fractional-order complex T system, the factional-order complex Lü system, and so forth.

In this article, we discuss the adaptive anti-synchronization between two different fractional-order chaotic complex systems described by a unified mathematical expression. Anti-synchronization error system is defined as $e(t)=y(t)+x(t)$, namely, $e^{r}(t)=y^{r}(t)+x^{r}(t)$ and $e^{i}(t)=y^{i}(t)+x^{i}(t)$. The main idea of this paper is to design the adaptive controller and the parameter update laws such that anti-synchronization error vector $e(t)$ converges to zero as $t \rightarrow \infty$, i.e., $\lim _{t \rightarrow \infty}\|e(t)\|=0$.

\section{Adaptive anti-synchronization schemes}

\subsection{The drive system without unknown parameters}

In this subsection, we consider two cases with different situations of B in the response system (2.1), i.e., $B$ is a real vector or a complex vector. Let us start with the first case in which B is a real vector.

Theorem 3.1. If the control law is designed as

$$
\begin{aligned}
U & =U^{r}+j U^{i}=-G(y) \hat{B}-g(y)-h(x)+K e \\
& =-G^{r}(y) \hat{B}-g^{r}(y)-h^{r}(x)+K e^{r}-j\left(G^{i}(y) \hat{B}+g^{i}(y)+h^{i}(x)-K e^{i}\right),
\end{aligned}
$$

and the adaptive laws are chosen as

$$
\left\{\begin{array}{l}
D_{*}^{\alpha} \hat{B}=\left(G^{r}(y)\right)^{\top} e^{r}+\left(G^{i}(y)\right)^{\top} e^{i}, \\
D_{*}^{\alpha} k_{l}=-\delta_{l}\left(\left(e_{l}^{r}(t)\right)^{2}+\left(e_{l}^{i}(t)\right)^{2}\right), \quad l=1,2, \ldots, n,
\end{array}\right.
$$

then anti-synchronization between systems (2.1) and (2.2) can be achieved, where $\hat{\mathrm{B}}$ denotes the estimated value of the unknown parameter vector $\mathrm{B}, \mathrm{K}=\operatorname{diag}\left(\mathrm{k}_{1}, \mathrm{k}_{2}, \ldots, \mathrm{k}_{\mathrm{n}}\right)$ represents the real control strength matrix, $\delta=$ $\operatorname{diag}\left(\delta_{1}, \delta_{2}, \ldots, \delta_{n}\right)$ is a convergence factor matrix with $\delta_{l}>0(l=1,2, \ldots, n)$.

Proof. It follows from the fractional-order chaotic complex systems (2.1) and (2.2) that

$$
D_{*}^{\alpha} e(t)=D_{*}^{\alpha} e^{r}(t)+j D_{*}^{\alpha} e^{i}(t)=G^{r}(y) B+g^{r}(y)+U^{r}+h^{r}(x)+j\left(G^{i}(y) B+g^{i}(y)+U^{i}+h^{i}(x)\right) .
$$

Thus, inserting (3.1) into (3.3) and separating real and imaginary parts, we obtain the error system as follows:

$$
\left\{\begin{array}{l}
D_{*}^{\alpha} e^{r}(t)=G^{r}(y) \tilde{B}+K e^{r} \\
D_{*}^{\alpha} e^{i}(t)=G^{i}(y) \tilde{B}+K e^{i},
\end{array}\right.
$$

where $\tilde{B}=B-\hat{B}=\left(\tilde{b}_{1}, \tilde{b}_{2}, \ldots, \tilde{b}_{\mathfrak{n}}\right)$ denotes the parameter estimation errors, $e^{r}(t)=\left(e_{1}^{r}(t), e_{2}^{r}(t), \ldots, e_{n}^{r}(t)\right)^{\top}$, and $e^{\mathfrak{i}}(\mathrm{t})=\left(e_{1}^{\mathfrak{i}}(\mathrm{t}), e_{2}^{\mathfrak{i}}(\mathrm{t}), \ldots, e_{\mathfrak{n}}^{\mathfrak{i}}(\mathrm{t})\right)^{\mathrm{T}}$. Furthermore, one has

$$
\left\{\begin{array}{l}
D_{*}^{\alpha} e_{l}^{r}(t)=G_{l}^{r}(y) \tilde{B}+k_{l} e_{l}^{r}, \\
D_{*}^{\alpha} e_{l}^{i}(t)=G_{l}^{i}(y) \tilde{B}+k_{l} e_{l}^{i},
\end{array}\right.
$$

where $G_{l}^{r}(y)$ and $G_{l}^{i}(y)$ are the l-th row of $G^{r}(y)$ and $G^{i}(y)$, respectively. Now, we consider a positive definite Lyapunov candidate function in the form

$$
V(e, t)=\frac{1}{2} \sum_{l=1}^{n}\left(\left(e_{l}^{r}(t)\right)^{2}+\left(e_{l}^{i}(t)\right)^{2}\right)+\frac{1}{2} \sum_{l=1}^{n} \frac{1}{\delta_{l}}\left(k_{l}+L\right)^{2}+\frac{1}{2} \tilde{B}^{T} \tilde{B},
$$

where $\mathrm{L}$ is a positive constant. According to Lemmas 2.2 and 2.3 , it is not difficult to obtain that

$$
D_{*}^{\alpha} V \leqslant \sum_{l=1}^{n}\left(e_{l}^{r} D_{*}^{\alpha} e_{l}^{r}+e_{l}^{i} D_{*}^{\alpha} e_{l}^{i}\right)+\sum_{l=1}^{n} \frac{1}{\delta_{l}}\left(k_{l}+L\right) D_{*}^{\alpha} k_{l}+\tilde{B}^{\top} D_{*}^{\alpha} \tilde{B} .
$$


In the case of (3.2) and (3.4), the relation (3.5) becomes

$$
\begin{aligned}
D_{*}^{\alpha} V & \leqslant \sum_{l=1}^{n}\left(e_{l}^{r}\left(G_{l}^{r}(y) \tilde{B}+k_{l} e_{l}^{r}\right)+e_{l}^{i}\left(G_{l}^{i}(y) \tilde{B}+k_{l} e_{l}^{i}\right)\right)+\tilde{B}^{T} D_{*}^{\alpha} \tilde{B}-\sum_{l=1}^{n}\left(k_{l}+L\right)\left(\left(e_{l}^{r}\right)^{2}+\left(e_{l}^{i}\right)^{2}\right) \\
& =\sum_{l=1}^{n}\left(e_{l}^{r} G_{l}^{r}(y) \tilde{B}+e_{l}^{i} G_{l}^{i}(y) \tilde{B}\right)-L \sum_{l=1}^{n}\left(\left(e_{l}^{r}\right)^{2}+\left(e_{l}^{i}\right)^{2}\right)+\tilde{B}^{\top} D_{*}^{\alpha} \tilde{B} .
\end{aligned}
$$

On the other hand,

$$
\begin{aligned}
& \sum_{l=1}^{n}\left(e_{l}^{r} G_{l}^{r}(y) \tilde{B}+e_{l}^{i} G_{l}^{i}(y) \tilde{B}\right) \\
& =\left[G_{1}^{r}(y) \tilde{B}, G_{2}^{r}(y) \tilde{B}, \ldots, G_{n}^{r}(y) \tilde{B}\right]\left[\begin{array}{c}
e_{1}^{r} \\
e_{2}^{r} \\
\vdots \\
e_{n}^{r}
\end{array}\right]+\left[G_{1}^{i}(y) \tilde{B}, G_{2}^{i}(y) \tilde{B}, \ldots, G_{n}^{i}(y) \tilde{B}\right]\left[\begin{array}{c}
e_{1}^{i} \\
e_{2}^{i} \\
\vdots \\
e_{n}^{i}
\end{array}\right] \\
& =\tilde{B}^{\top}\left[\left(G_{1}^{r}(y)\right)^{\top},\left(G_{2}^{r}(y)\right)^{\top}, \ldots,\left(G_{n}^{r}(y)\right)^{\top}\right]\left[\begin{array}{c}
e_{1}^{r} \\
e_{2}^{r} \\
\vdots \\
e_{n}^{r}
\end{array}\right]+\tilde{B}^{\top}\left[\left(G_{1}^{i}(y)\right)^{\top},\left(G_{2}^{i}(y)\right)^{\top}, \ldots,\left(G_{n}^{i}(y)\right)^{\top}\right]\left[\begin{array}{c}
e_{1}^{i} \\
e_{2}^{i} \\
\vdots \\
e_{n}^{i}
\end{array}\right] \\
& =\tilde{B}^{\top}\left[\left(G^{r}(y)\right)^{\top},\left(G^{i}(y)\right)^{\top}\right]\left[\begin{array}{c}
e^{r} \\
e^{i}
\end{array}\right]=\tilde{B}^{\top}\left(\left(G^{r}(y)\right)^{\top} e^{r}+\left(G^{i}(y)\right)^{\top} e^{i}\right) .
\end{aligned}
$$

Thus, combining (3.2) and the latter equality implies that

$$
D_{*}^{\alpha} V \leqslant-L\left(\left(e^{r}\right)^{\top} e^{r}+\left(e^{i}\right)^{\top} e^{i}\right)
$$

From Lemma 2.4, we deduce that

$$
\lim _{t \rightarrow \infty}\left\|e^{r}(t)\right\|=0 \text { and } \quad \lim _{t \rightarrow \infty}\left\|e^{i}(t)\right\|=0 .
$$

Hence, we have $\lim _{t \rightarrow \infty}\|e(t)\|=0$. Therefore, the designed controller (3.1) and the fractional adaption laws (3.2) enable the drive system (2.2) and the response system (2.1) to achieve anti-synchronization. The proof is complete.

Now, we turn our attention to the case when B is a complex vector. Then B can be rewritten as $\mathrm{B}=\mathrm{B}^{\mathrm{r}}+j \mathrm{~B}^{\mathrm{i}}$ and the fractional-order system (2.1) can be represented as

$$
D_{*}^{\alpha} y=G(y) B^{r}+j G(y) B^{i}+g(y)+U=G(y) B^{r}+Q(y) B^{i}+g(y)+U,
$$

where $Q(y)=j G(y)$ describes a new $n \times n$ complex matrix. By the same procedure, we obtain the following result.

Theorem 3.2. If the control law is designed as

$$
\begin{aligned}
U & =U^{r}+j U^{i}=-G(y) \hat{B}^{r}-Q(y) \hat{B}^{i}-g(y)-h(x)+K e \\
& =-G^{r}(y) \hat{B}^{r}-Q^{r}(y) \hat{B}^{i}-g^{r}(y)-h^{r}(x)+K e^{r}-j\left(G^{i}(y) \hat{B}^{r}+Q^{i}(y) \hat{B}^{i}+g^{i}(y)+h^{i}(x)-K e^{i}\right),
\end{aligned}
$$

and the adaptive laws are chosen as

$$
\left\{\begin{array}{l}
D_{*}^{\alpha} \hat{B}^{r}=\left(G^{r}(y)\right)^{\top} e^{r}+\left(G^{i}(y)\right)^{\top} e^{i}, \\
D_{*}^{\alpha} \hat{B}^{i}=\left(Q^{r}(y)\right)^{\top} e^{r}+\left(Q^{i}(y)\right)^{\top} e^{i}, \\
D_{*}^{\alpha} k_{l}=-\delta_{l}\left(\left(e_{l}^{r}(t)\right)^{2}+\left(e_{l}^{i}(t)\right)^{2}\right), \quad l=1,2, \ldots, n,
\end{array}\right.
$$


then anti-synchronization between systems (2.2) and (3.6) can be achieved, where $\hat{\mathrm{B}}^{\mathrm{r}}$ and $\hat{\mathrm{B}}^{\mathrm{i}}$ denote the estimated values of the unknown parameter vectors $\mathrm{B}^{\mathrm{r}}$ and $\mathrm{B}^{\mathrm{i}}$, respectively, $\mathrm{K}=\operatorname{diag}\left(\mathrm{k}_{1}, \mathrm{k}_{2}, \ldots, \mathrm{k}_{\mathrm{n}}\right)$ represents the real control strength matrix, $\delta=\operatorname{diag}\left(\delta_{1}, \delta_{2}, \ldots, \delta_{n}\right)$ is a convergence factor matrix with $\delta_{l}>0(l=1,2, \ldots, n)$.

Proof. The proof is analogous to that of Theorem 3.1, and hence is omitted.

\subsection{The drive system with unknown parameters}

In this subsection, we consider the case where the drive system has unknown parameters. If some unknown parameters exist in the drive system (2.2), then system (2.2) can be written as

$$
D_{*}^{\alpha} x=F(x) A+f(x)
$$

where $x=\left(x_{1}, x_{2}, \ldots, x_{n}\right)^{\top} \in \mathbb{C}^{n}$ denotes the complex state vector, $F(x) \in \mathbb{C}^{n \times n}$ is a complex matrix, $A=\left(a_{1}, a_{2}, \ldots, a_{n}\right)^{\top}$ represents the real (or complex) vector of unknown parameters, and $f: \mathbb{C}^{n} \rightarrow \mathbb{C}^{n}$ is a nonlinear complex vector function.

In what follows, we consider the case when $A$ and $B$ are real vectors.

Theorem 3.3. If the control law is selected as follows:

$$
\begin{aligned}
U & =U^{r}+j U^{i}=-G(y) \hat{B}-g(y)-F(x) \hat{A}-f(x)+K e \\
& =-G^{r}(y) \hat{B}-g^{r}(y)-F^{r}(x) \hat{A}-f^{r}(x)+K e^{r}-j\left(G^{i}(y) \hat{B}+g^{i}(y)+F^{i}(x) \hat{A}+f^{i}(x)-K e^{i}\right),
\end{aligned}
$$

and the adaptive laws are chosen as

$$
\left\{\begin{array}{l}
D_{*}^{\alpha} \hat{A}=\left(F^{r}(x)\right)^{\top} e^{r}+\left(F^{i}(x)\right)^{\top} e^{i} \\
D_{*}^{\alpha} \hat{B}=\left(G^{r}(y)\right)^{\top} e^{r}+\left(G^{i}(y)\right)^{\top} e^{i}, \\
D_{*}^{\alpha} k_{l}=-\delta_{l}\left(\left(e_{l}^{r}(t)\right)^{2}+\left(e_{l}^{i}(t)\right)^{2}\right), \quad l=1,2, \ldots, n,
\end{array}\right.
$$

then the response system (2.1) can anti-synchronize the drive system (3.7), where $\hat{A}$ and $\hat{B}$ denote the estimated values of the unknown parameter vectors $\mathrm{A}$ and $\mathrm{B}$, respectively, $\mathrm{K}=\operatorname{diag}\left(\mathrm{k}_{1}, \mathrm{k}_{2}, \ldots, \mathrm{k}_{\mathrm{n}}\right)$ is the real control strength matrix, $\delta=\operatorname{diag}\left(\delta_{1}, \delta_{2}, \ldots, \delta_{n}\right)$ is a convergence factor matrix with $\delta_{l}>0(l=1,2, \ldots, n)$.

Proof. From the fractional-order chaotic complex systems (2.1), (3.7), and the controller (3.8), the error dynamical system can be obtained as follows:

$$
D_{*}^{\alpha} e(t)=D_{*}^{\alpha} y(t)+D_{*}^{\alpha} x(t)=G(y) B+g(y)+U+F(x) A+f(x)=F(x) \tilde{A}+G(y) \tilde{B}+K e,
$$

where $\tilde{A}=A-\hat{A}=\left(\tilde{a}_{1}, \tilde{a}_{2}, \ldots, \tilde{a}_{n}\right), \tilde{B}=B-\hat{B}=\left(\tilde{b}_{1}, \tilde{b}_{2}, \ldots, \tilde{b}_{n}\right)$ are the errors between the true values and estimated values of unknown parameters. Thus, separating real and imaginary parts of (3.10) gives the error system as follows:

$$
\left\{\begin{array}{l}
D_{*}^{\alpha} e^{r}(t)=F^{r}(x) \tilde{A}+G^{r}(y) \tilde{B}+K e^{r} \\
D_{*}^{\alpha} e^{i}(t)=F^{i}(x) \tilde{A}+G^{i}(y) \tilde{B}+K e^{i},
\end{array}\right.
$$

where $e^{r}(t)=\left(e_{1}^{r}(t), e_{2}^{r}(t), \ldots, e_{n}^{r}(t)\right)^{\top}$ and $e^{i}(t)=\left(e_{1}^{i}(t), e_{2}^{i}(t), \ldots, e_{n}^{i}(t)\right)^{\top}$. Moreover, we have

$$
\left\{\begin{array}{l}
D_{*}^{\alpha} e_{l}^{r}(t)=F_{l}^{r}(x) \tilde{A}+G_{l}^{r}(y) \tilde{B}+k_{l} e_{l}^{r}, \\
D_{*}^{\alpha} e_{l}^{i}(t)=F_{l}^{i}(x) \tilde{A}+G_{l}^{i}(y) \tilde{B}+k_{l} e_{l}^{i},
\end{array}\right.
$$

where $F_{l}^{r}(x), F_{l}^{i}(x), G_{l}^{r}(y)$, and $G_{l}^{i}(y)$ are the l-th row of $F^{r}(x), F^{i}(x), G^{r}(y)$, and $G^{i}(y)$, respectively. Define a positive definite Lyapunov candidate function as follows:

$$
V(e, t)=\frac{1}{2} \sum_{l=1}^{n}\left(\left(e_{l}^{r}(t)\right)^{2}+\left(e_{l}^{i}(t)\right)^{2}\right)+\frac{1}{2} \sum_{l=1}^{n} \frac{1}{\delta_{l}}\left(k_{l}+L\right)^{2}+\frac{1}{2} \tilde{A}^{\top} \tilde{A}+\frac{1}{2} \tilde{B}^{\top} \tilde{B},
$$


where $L$ is a positive constant. Then,

$$
\begin{aligned}
D_{*}^{\alpha} V \leqslant & \sum_{l=1}^{n}\left(e_{l}^{r} D_{*}^{\alpha} e_{l}^{r}+e_{l}^{i} D_{*}^{\alpha} e_{l}^{i}\right)+\sum_{l=1}^{n} \frac{1}{\delta_{l}}\left(k_{l}+L\right) D_{*}^{\alpha} k_{l}+\tilde{A}^{\top} D_{*}^{\alpha} \tilde{A}+\tilde{B}^{\top} D_{*}^{\alpha} \tilde{B} \\
\leqslant & \sum_{l=1}^{n}\left(e_{l}^{r}\left(F_{l}^{r}(x) \tilde{A}+G_{l}^{r}(y) \tilde{B}+k_{l} e_{l}^{r}\right)\right)+\sum_{l=1}^{n}\left(e_{l}^{i}\left(F_{l}^{i}(x) \tilde{A}+G_{l}^{i}(y) \tilde{B}+k_{l} e_{l}^{i}\right)\right) \\
& -\sum_{l=1}^{n}\left(k_{l}+L\right)\left(\left(e_{l}^{r}\right)^{2}+\left(e_{l}^{i}\right)^{2}\right)+\tilde{A}^{\top} D_{*}^{\alpha} \tilde{A}+\tilde{B}^{\top} D_{*}^{\alpha} \tilde{B} \\
= & \sum_{l=1}^{n}\left(e_{l}^{r} F_{l}^{r}(x) \tilde{A}+e_{l}^{i} F_{l}^{i}(x) \tilde{A}\right)+\sum_{l=1}^{n}\left(e_{l}^{r} G_{l}^{r}(y) \tilde{B}+e_{l}^{i} G_{l}^{i}(y) \tilde{B}\right)+\tilde{A}^{\top} D_{*}^{\alpha} \tilde{A} \\
& +\tilde{B}^{\top} D_{*}^{\alpha} \tilde{B}-L \sum_{l=1}^{n}\left(\left(e_{l}^{r}\right)^{2}+\left(e_{l}^{i}\right)^{2}\right) .
\end{aligned}
$$

From the adaptive laws (3.9) and

$$
\left\{\begin{array}{l}
\sum_{l=1}^{n}\left(e_{l}^{r} F_{l}^{r}(x) \tilde{A}+e_{l}^{i} F_{l}^{i}(x) \tilde{A}\right)=\tilde{A}^{\top}\left(\left(F^{r}(x)\right)^{\top} e^{r}+\left(F^{i}(x)\right)^{\top} e^{i}\right), \\
\sum_{l=1}^{n}\left(e_{l}^{r} G_{l}^{r}(y) \tilde{B}+e_{l}^{i} G_{l}^{i}(y) \tilde{B}\right)=\tilde{B}^{\top}\left(\left(G^{r}(y)\right)^{\top} e^{r}+\left(G^{i}(y)\right)^{\top} e^{i}\right)
\end{array}\right.
$$

we conclude that

$$
D_{*}^{\alpha} V \leqslant-L\left(\left(e^{r}\right)^{\top} e^{r}+\left(e^{i}\right)^{\top} e^{i}\right)
$$

The rest of the proof is similar to that of Theorem 3.1 and so is omitted. This completes the proof.

Thus, as a consequence of Theorem 3.3, we can obtain the following corollary.

Corollary 3.4. If the structures of general fractional-order chaotic complex systems (2.1) and (3.7) are identical, i.e., $\mathrm{F}(\cdot)=\mathrm{G}(\cdot), \mathrm{f}(\cdot)=\mathrm{g}(\cdot)$, and $\mathrm{A}=\mathrm{B}$, the controller is designed as

$$
\begin{aligned}
\mathrm{U} & =\mathrm{U}^{\mathrm{r}}+j \mathrm{U}^{i}=-(\mathrm{F}(\mathrm{y})+\mathrm{F}(\mathrm{x})) \hat{A}-f(y)-f(x)+K e \\
& =-\left(F^{r}(y)+F^{r}(x)\right) \hat{A}-f^{r}(y)-f^{r}(x)+K e^{r}-j\left(\left(F^{i}(y)+F^{i}(x)\right) \hat{A}+f^{i}(y)+f^{i}(x)-K e^{i}\right),
\end{aligned}
$$

and the adaptive laws are chosen as

$$
\left\{\begin{array}{l}
D_{*}^{\alpha} \hat{A}=\left(F^{r}(x)\right)^{\top} e^{r}+\left(F^{i}(x)\right)^{\top} e^{i}, \\
D_{*}^{\alpha} k_{l}=-\delta_{l}\left(\left(e_{l}^{r}(t)\right)^{2}+\left(e_{l}^{i}(t)\right)^{2}\right), \quad l=1,2, \ldots, n,
\end{array}\right.
$$

then anti-synchronization of two identical general fractional-order chaotic complex systems is also achieved, where $\hat{A}$ is the estimated value of the unknown parameter vector $A, K=\operatorname{diag}\left(k_{1}, k_{2}, \ldots, k_{n}\right)$ is the real control strength matrix, $\delta=\operatorname{diag}\left(\delta_{1}, \delta_{2}, \ldots, \delta_{n}\right)$ is a convergence factor matrix with $\delta_{l}>0(l=1,2, \ldots, n)$.

In the following, we consider three cases with different situations of unknown parameters. If $A$ is a real vector and $B$ is a complex vector, then adaptive anti-synchronization scheme between systems (3.6) and (3.7) is obtained as follows.

Theorem 3.5. If the control law is selected as

$$
\begin{aligned}
U= & U^{r}+j U^{i}=-G(y) \hat{B}^{r}-Q(y) \hat{B}^{i}-g(y)-F(x) \hat{A}-f(x)+K e \\
= & -G^{r}(y) \hat{B}^{r}-Q^{r}(y) \hat{B}^{i}-g^{r}(y)-F^{r}(x) \hat{A}-f^{r}(x)+K e^{r} \\
& -j\left(G^{i}(y) \hat{B}^{r}+Q^{i}(y) \hat{B}^{i}+g^{i}(y)+F^{i}(x) \hat{A}+f^{i}(x)-K e^{i}\right),
\end{aligned}
$$


and the adaptive laws are chosen as

$$
\left\{\begin{array}{l}
D_{*}^{\alpha} \hat{A}=\left(F^{r}(x)\right)^{\top} e^{r}+\left(F^{i}(x)\right)^{\top} e^{i}, \\
D_{*}^{\alpha} \hat{B}^{r}=\left(G^{r}(y)\right)^{\top} e^{r}+\left(G^{i}(y)\right)^{\top} e^{i}, \quad D_{*}^{\alpha} \hat{B}^{i}=\left(Q^{r}(y)\right)^{\top} e^{r}+\left(Q^{i}(y)\right)^{\top} e^{i}, \\
D_{*}^{\alpha} k_{l}=-\delta_{l}\left(\left(e_{l}^{r}(t)\right)^{2}+\left(e_{l}^{i}(t)\right)^{2}\right), \quad l=1,2, \ldots, n,
\end{array}\right.
$$

then the response system (3.6) can anti-synchronize the drive system (3.7), where $\hat{\mathrm{A}}, \hat{\mathrm{B}}^{r}$, and $\hat{\mathrm{B}}^{i}$ are the estimated values of the unknown parameter vectors $A, B^{r}$, and $B^{i}$, respectively, $K=\operatorname{diag}\left(k_{1}, k_{2}, \ldots, k_{n}\right)$ denotes the real control strength matrix, $\delta=\operatorname{diag}\left(\delta_{1}, \delta_{2}, \ldots, \delta_{n}\right)$ is a convergence factor matrix with $\delta_{l}>0(l=1,2, \ldots, n)$.

Proof. The proof is similar to that of Theorem 3.3 and thus is omitted.

If $A$ is a complex vector and $B$ is a real vector, then the response system is described as (2.1) and the drive system (3.7) can be rewritten as

$$
D_{*}^{\alpha} x=F(x) A^{r}+j F(x) A^{i}+f(x)=F(x) A^{r}+P(x) A^{i}+f(x),
$$

where $P(x)=j F(x)$ is a new $n \times n$ complex matrix.

Theorem 3.6. If the control law is selected as follows:

$$
\begin{aligned}
u= & U^{r}+j U^{i}=-G(y) \hat{B}-g(y)-F(x) \hat{A}^{r}-P(x) \hat{A}^{i}-f(x)+K e \\
= & -G^{r}(y) \hat{B}-g^{r}(y)-F^{r}(x) \hat{A}^{r}-P^{r}(x) \hat{A}^{i}-f^{r}(x)+K e^{r} \\
& -j\left(G^{i}(y) \hat{B}+g^{i}(y)+F^{i}(x) \hat{A}^{r}+P^{i}(x) \hat{A}^{i}+f^{i}(x)-K e^{i}\right),
\end{aligned}
$$

and the adaptive laws are chosen as

$$
\begin{cases}D_{*}^{\alpha} \hat{A}^{r}=\left(F^{r}(x)\right)^{\top} e^{r}+\left(F^{i}(x)\right)^{\top} e^{i}, & D_{*}^{\alpha} \hat{A}^{i}=\left(P^{r}(x)\right)^{\top} e^{r}+\left(P^{i}(x)\right)^{\top} e^{i}, \\ D_{*}^{\alpha} \hat{B}=\left(G^{r}(y)\right)^{\top} e^{r}+\left(G^{i}(y)\right)^{\top} e^{i}, & \\ D_{*}^{\alpha} k_{l}=-\delta_{l}\left(\left(e_{l}^{r}(t)\right)^{2}+\left(e_{l}^{i}(t)\right)^{2}\right), & l=1,2, \ldots, n,\end{cases}
$$

then the response system (2.1) can anti-synchronize the drive system (3.11), where $\hat{A}^{r}, \hat{A}^{i}$, and $\hat{B}$ are the estimated values of the unknown parameter vectors $A^{r}, A^{i}$, and $B$, respectively, $K=\operatorname{diag}\left(k_{1}, k_{2}, \ldots, k_{n}\right)$ denotes the real control strength matrix, $\delta=\operatorname{diag}\left(\delta_{1}, \delta_{2}, \ldots, \delta_{n}\right)$ is a convergence factor matrix with $\delta_{l}>0(l=1,2, \ldots, n)$.

Proof. The proof is similar to that of Theorem 3.5, and so is omitted.

When $A$ and $B$ are complex vectors, the response system is equivalent to (3.6) and the drive system is (3.11). Then we have the following anti-synchronization scheme between systems (3.6) and (3.11).

Theorem 3.7. If the control law is selected as follows:

$$
U=-G(y) \hat{B}^{r}-Q(y) \hat{B}^{i}-g(y)-F(x) \hat{A}^{r}-P(x) \hat{A}^{i}-f(x)+K e,
$$

and the adaptive laws are chosen as

$$
\left\{\begin{array}{l}
D_{*}^{\alpha} \hat{A}^{r}=\left(F^{r}(x)\right)^{\top} e^{r}+\left(F^{i}(x)\right)^{\top} e^{i}, \quad D_{*}^{\alpha} \hat{A}^{i}=\left(P^{r}(x)\right)^{\top} e^{r}+\left(P^{i}(x)\right)^{\top} e^{i}, \\
D_{*}^{\alpha} \hat{B}^{r}=\left(G^{r}(y)\right)^{\top} e^{r}+\left(G^{i}(y)\right)^{\top} e^{i}, \quad D_{*}^{\alpha} \hat{B}^{i}=\left(Q^{r}(y)\right)^{\top} e^{r}+\left(Q^{i}(y)\right)^{\top} e^{i}, \\
D_{*}^{\alpha} k_{l}=-\delta_{l}\left(\left(e_{l}^{r}(t)\right)^{2}+\left(e_{l}^{i}(t)\right)^{2}\right), \quad l=1,2, \ldots, n,
\end{array}\right.
$$

then the response system (3.6) can anti-synchronize the drive system (3.11), where $\hat{\mathrm{A}}^{r}, \hat{\mathrm{A}}^{i}, \hat{\mathrm{B}}^{r}$, and $\hat{\mathrm{B}}^{i}$ are the estimated values of the unknown parameter vectors $A^{r}, A^{i}, B^{r}$, and $B^{i}$, respectively, $K=\operatorname{diag}\left(k_{1}, k_{2}, \ldots, k_{n}\right)$ denotes the real control strength matrix, $\delta=\operatorname{diag}\left(\delta_{1}, \delta_{2}, \ldots, \delta_{n}\right)$ is a convergence factor matrix with $\delta_{l}>0$ $(l=1,2, \ldots, n)$.

Proof. The proof is similar to that of Theorem 3.3, and therefore is omitted. 


\section{Numerical simulations}

In order to illustrate anti-synchronization between two different fractional-order chaotic complex systems with unknown parameters from the point of theoretical view, it is assumed that the fractional-order complex Lorenz system drives the fractional-order complex Lü system. Thus, the drive system is introduced in the form as

$$
\left\{\begin{array}{l}
D_{*}^{\alpha} x_{1}=a_{1}\left(x_{2}-x_{1}\right), \\
D_{*}^{\alpha} x_{2}=a_{2} x_{1}-x_{2}-x_{1} x_{3}, \\
D_{*}^{\alpha} x_{3}=\frac{1}{2}\left(\bar{x}_{1} x_{2}+x_{1} \bar{x}_{2}\right)-a_{3} x_{3},
\end{array}\right.
$$

where $x_{1}=m_{1}+j m_{2}, x_{2}=m_{3}+j m_{4}$ are complex state variables, $x_{3}=m_{5}$ is a real state variable, and

$$
\begin{aligned}
& F(x)=\left(\begin{array}{ccc}
x_{2}-x_{1} & 0 & 0 \\
0 & x_{1} & 0 \\
0 & 0 & -x_{3}
\end{array}\right)=\left(\begin{array}{ccc}
m_{3}-m_{1} & 0 & 0 \\
0 & m_{1} & 0 \\
0 & 0 & -m_{5}
\end{array}\right)+j\left(\begin{array}{ccc}
m_{4}-m_{2} & 0 & 0 \\
0 & m_{2} & 0 \\
0 & 0 & 0
\end{array}\right) \\
& f(x)=\left(\begin{array}{c}
0 \\
-x_{2}-x_{1} x_{3} \\
\frac{1}{2}\left(\bar{x}_{1} x_{2}+x_{1} \bar{x}_{2}\right)
\end{array}\right)=\left(\begin{array}{c}
0 \\
-m_{3}-m_{1} m_{5} \\
\left.m_{1} m_{3}+m_{2} m_{4}\right)
\end{array}\right)+j\left(\begin{array}{c}
0 \\
-m_{4}-m_{2} m_{5} \\
0
\end{array}\right), \quad A=\left(\begin{array}{l}
a_{1} \\
a_{2} \\
a_{3}
\end{array}\right) .
\end{aligned}
$$

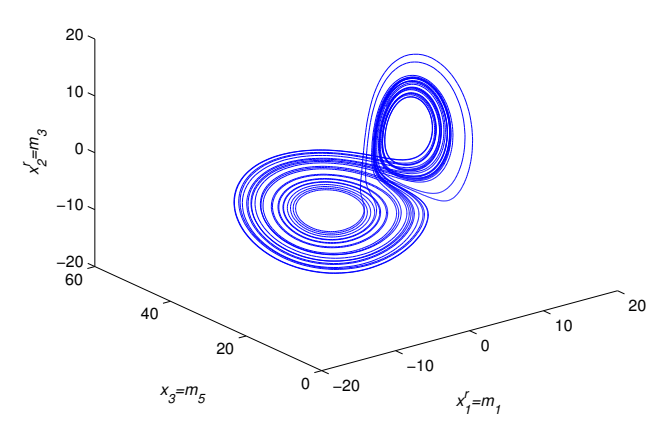

(a)

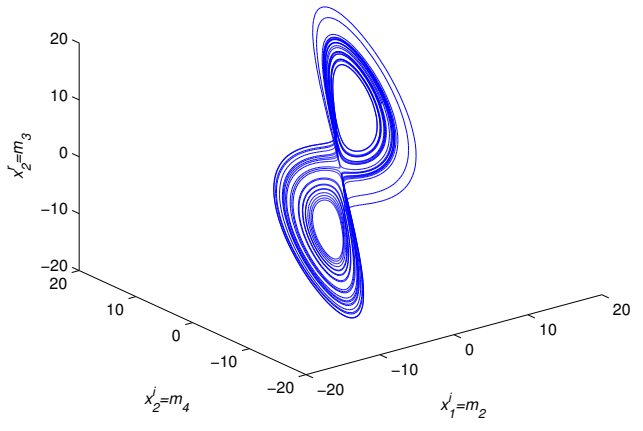

(b)

Figure 1: Chaotic attractors of the fractional-order complex Lorenz system (4.1) in different projections (a) $\left(x_{1}^{r}, x_{3}, x_{2}^{r}\right)$ and (b) $\left(x_{1}^{i}, x_{2}^{i}, x_{2}^{r}\right)$.

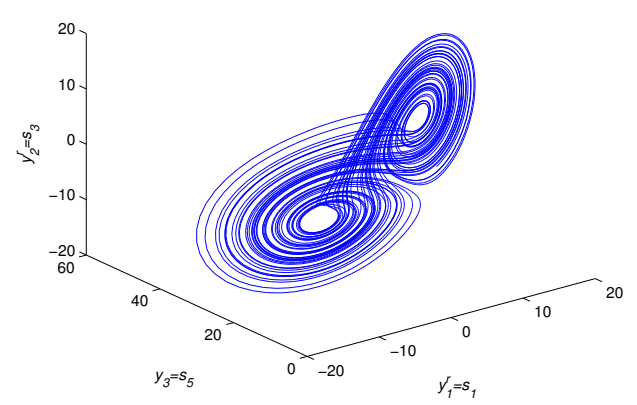

(a)

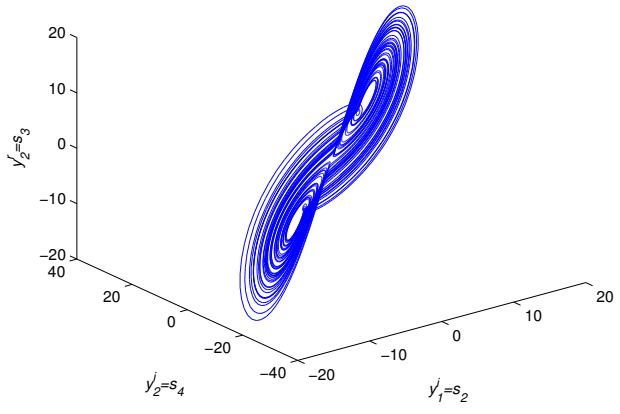

(b)

Figure 2: Chaotic attractors of the fractional-order complex Lü system (4.2) in different projections (a) $\left(y_{1}^{r}, y_{3}, y_{2}^{r}\right)$ and (b) $\left(y_{1}^{i}\right.$, $\left.y_{2}^{i}, y_{2}^{r}\right)$. 


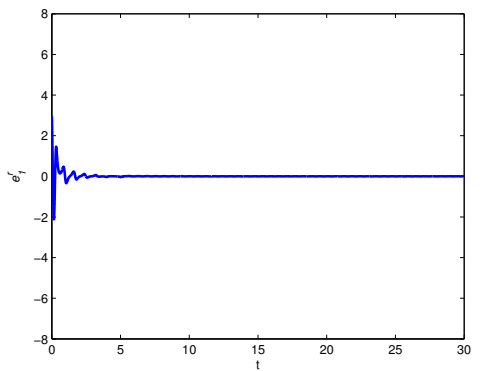

(a)

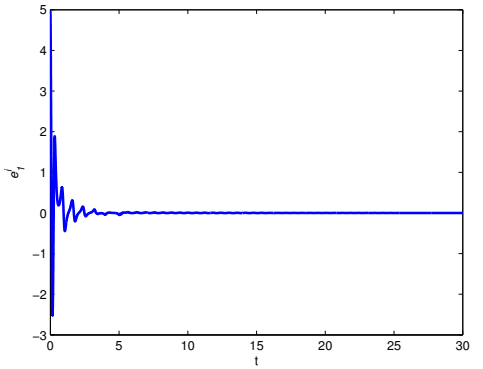

(b)

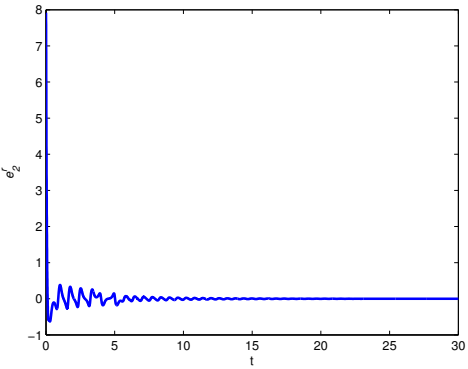

(c)

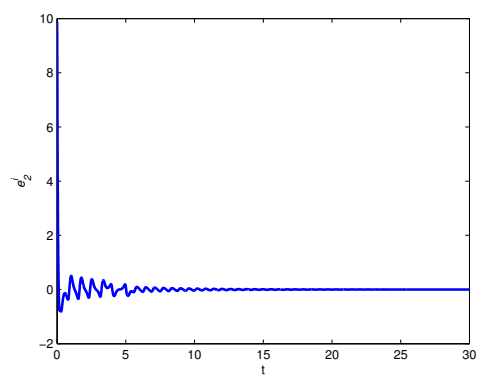

(d)

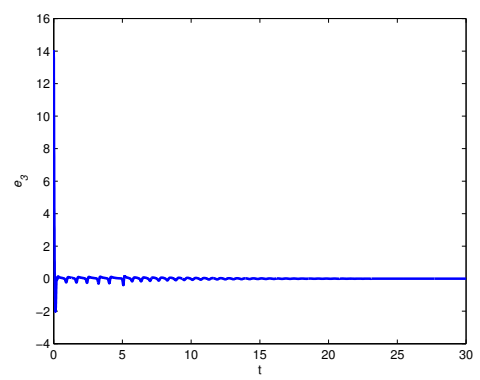

(e)

Figure 3: The error dynamics of anti-synchronization between two different fractional-order chaotic complex systems (4.1) and (4.2) with $\delta_{1}=8, \delta_{2}=5$, and $\delta_{3}=6$.

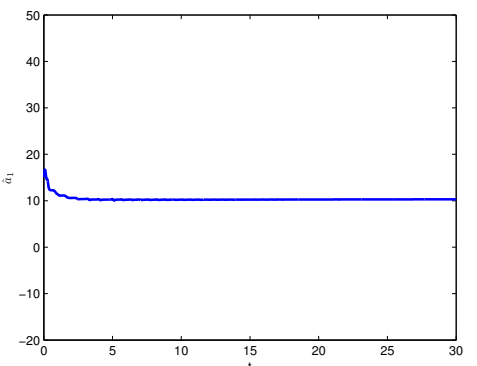

(a)

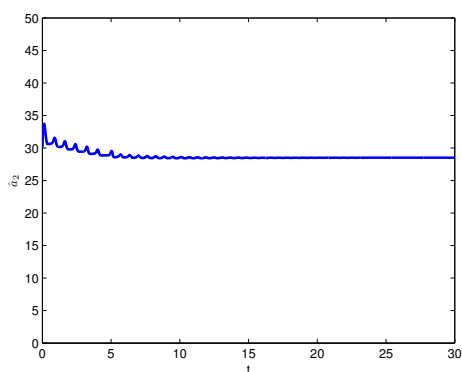

(b)

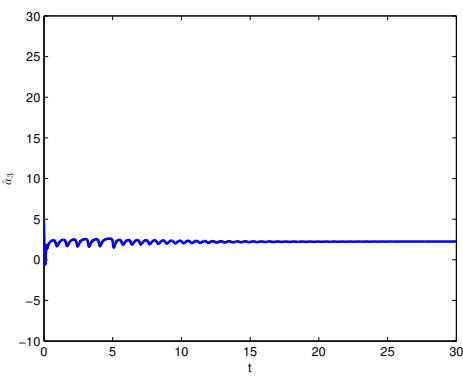

(c)

Figure 4: Changes of the parameters $\hat{a}_{1}, \hat{a}_{2}, \hat{a}_{3}$ with time $t$.

The response system is described as

$$
\left\{\begin{array}{l}
D_{*}^{\alpha} y_{1}=b_{1}\left(y_{2}-y_{1}\right)+u_{1} \\
D_{*}^{\alpha} y_{2}=-y_{1} y_{3}+b_{2} y_{2}+u_{2} \\
D_{*}^{\alpha} y_{3}=\frac{1}{2}\left(\bar{y}_{1} y_{2}+y_{1} \bar{y}_{2}\right)-b_{3} y_{3}+u_{3}
\end{array}\right.
$$

where $y_{1}=s_{1}+j s_{2}, y_{2}=s_{3}+j s_{4}$ are complex state variables, $y_{3}=s_{5}$ is a real state variable, $u_{1}=u_{1}+j u_{2}$, $\mathrm{u}_{2}=\mathrm{u}_{3}+j \mathrm{u}_{4}, \mathrm{u}_{3}=\mathrm{u}_{5}$ are control functions to be determined in the later discussion, and

$$
G(y)=\left(\begin{array}{ccc}
y_{2}-y_{1} & 0 & 0 \\
0 & y_{2} & 0 \\
0 & 0 & -y_{3}
\end{array}\right)=\left(\begin{array}{ccc}
s_{3}-s_{1} & 0 & 0 \\
0 & s_{3} & 0 \\
0 & 0 & -s_{5}
\end{array}\right)+j\left(\begin{array}{ccc}
s_{4}-s_{2} & 0 & 0 \\
0 & s_{4} & 0 \\
0 & 0 & 0
\end{array}\right),
$$




$$
g(y)=\left(\begin{array}{c}
0 \\
-y_{1} y_{3} \\
\frac{1}{2}\left(\bar{y}_{1} y_{2}+y_{1} \bar{y}_{2}\right)
\end{array}\right)=\left(\begin{array}{c}
0 \\
-s_{1} s_{5} \\
\left.s_{1} s_{3}+s_{2} s_{4}\right)
\end{array}\right)+j\left(\begin{array}{c}
0 \\
-s_{2} s_{5} \\
0
\end{array}\right), \quad B=\left(\begin{array}{l}
b_{1} \\
b_{2} \\
b_{3}
\end{array}\right) .
$$

According to Theorem 3.3, the controller is designed as

$$
\left\{\begin{array}{l}
u_{1}=-\hat{b}_{1}\left(s_{3}-s_{1}\right)-\hat{a}_{1}\left(m_{3}-m_{1}\right)+k_{1} e_{1}^{r} \\
u_{2}=-\hat{b}_{1}\left(s_{4}-s_{2}\right)-\hat{a}_{1}\left(m_{4}-m_{2}\right)+k_{1} e_{1}^{i} \\
u_{3}=s_{1} s_{5}-\hat{b}_{2} s_{3}+m_{3}+m_{1} m_{5}-\hat{a}_{2} m_{1}+k_{2} e_{2}^{r}, \\
u_{4}=s_{2} s_{5}-\hat{b}_{2} s_{4}+m_{4}+m_{2} m_{5}-\hat{a}_{2} m_{2}+k_{2} e_{2}^{i} \\
u_{5}=-s_{1} s_{3}-s_{2} s_{4}+\hat{b}_{3} s_{5}-m_{1} m_{3}-m_{2} m_{4}+\hat{a}_{3} m_{5}+k_{3} e_{3},
\end{array}\right.
$$

and the adaptive laws of parameters are chosen as

$$
\begin{aligned}
& \left\{\begin{array}{l}
D_{*}^{\alpha} \hat{a}_{1}=\left(m_{3}-m_{1}\right) e_{1}^{r}+\left(m_{4}-m_{2}\right) e_{1}^{i}, \\
D_{*}^{\alpha} \hat{a}_{2}=m_{1} e_{2}^{r}+m_{2} e_{2}^{i}, \\
D_{*}^{\alpha} \hat{a}_{3}=-m_{5} e_{3},
\end{array}\right. \\
& \left\{\begin{array}{l}
D_{*}^{\alpha} \hat{b}_{1}=\left(s_{3}-s_{1}\right) e_{1}^{r}+\left(s_{4}-s_{2}\right) e_{1}^{i}, \\
D_{*}^{\alpha} \hat{b}_{2}=s_{3} e_{2}^{r}+s_{4} e_{2}^{i} \\
D_{*}^{\alpha} \hat{b}_{3}=-s_{5} e_{3}
\end{array}\right. \\
& \left\{\begin{array}{l}
D_{*}^{\alpha} k_{1}=-\delta_{1}\left[\left(e_{1}^{r}\right)^{2}+\left(e_{1}^{i}\right)^{2}\right], \\
D_{*}^{\alpha} k_{2}=-\delta_{2}\left[\left(e_{2}^{r}\right)^{2}+\left(e_{2}^{i}\right)^{2}\right] \\
D_{*}^{\alpha} k_{3}=-\delta_{3} e_{3}^{2} .
\end{array}\right.
\end{aligned}
$$

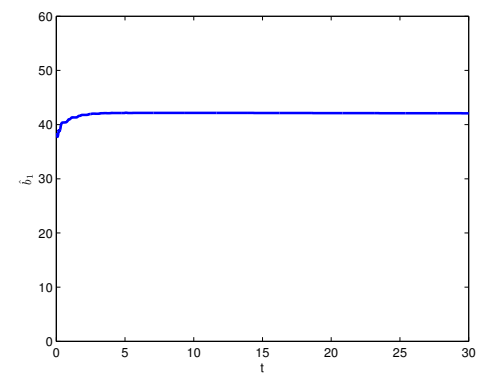

(a)

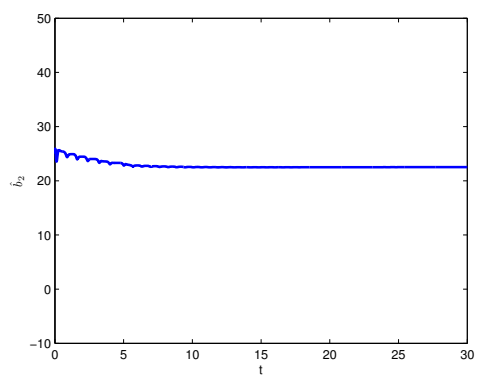

(b)

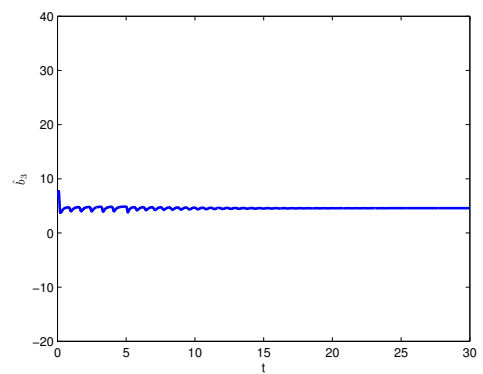

(c)

Figure 5: Changes of the parameters $\hat{b}_{1}, \hat{b}_{2}, \hat{b}_{3}$ with time $t$.

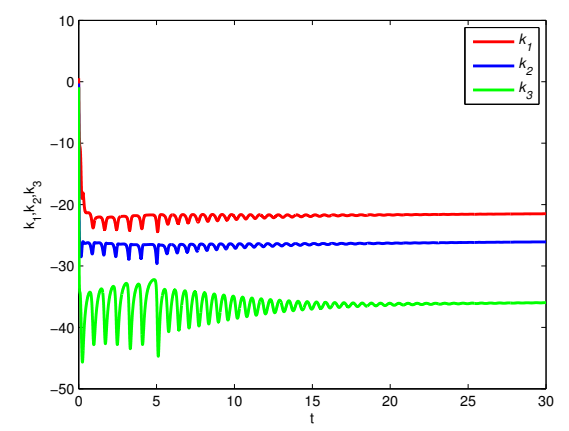

Figure 6: Time evolution of the control coefficients $k_{1}, k_{2}$, and $k_{3}$. 
In the numerical simulations, let $\alpha=0.998$ and the true values of the unknown parameters be chosen as $A=(10,28,8 / 3)^{\mathrm{T}}$ and $B=(42,22,5)^{\mathrm{T}}$. The initial values of the drive and response systems are chosen as $x(0)=(2+3 j, 5+6 j, 9)^{\top}$ and $y(0)=(1+2 j, 3+4 j, 5)^{\top}$, respectively. When we select the above system parameters and fractional orders, systems (4.1) and (4.2) without the controller can generate chaotic attractors, see Figures 1 and 2, respectively. In addition, we choose initial values of the estimated parameters and control strength as $\hat{A}(0)=(15,30,5)^{\top}, \hat{B}(0)=(38,25,8)^{\top}$, and $\mathrm{K}=\operatorname{diag}(1,1,1)$. The results on adaptive anti-synchronization between (4.1) and (4.2) are shown in Figures 3-6. The errors of anti-synchronization converge asymptotically to zero, as demonstrated in Figure 3. Figures 4 and 5 display the estimates $\hat{a}_{1}, \hat{a}_{2}, \hat{a}_{3}$ and $\hat{b}_{1}, \hat{b}_{2}, \hat{b}_{3}$ of the unknown parameters. From Figure 6 , it is clear that the control coefficients $k_{l}(l=1,2,3)$ converge to some constants as $t \rightarrow \infty$, respectively. As expected, the above results demonstrate the anti-synchronization has been achieved between two fractional-order chaotic complex systems (4.1) and (4.2) with unknown parameters.

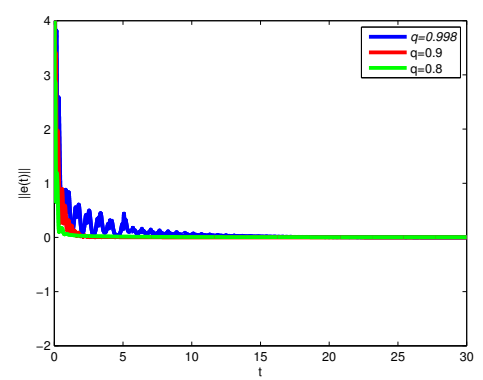

(a) The system errors of different fractional.

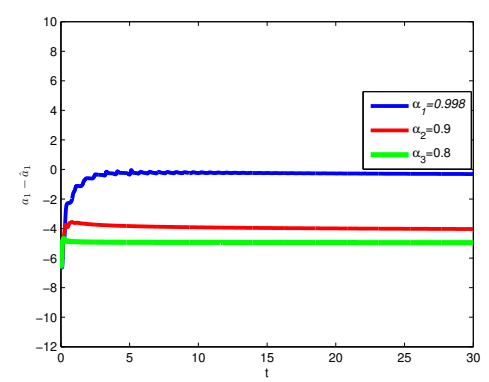

(b) The error $a_{1}-\hat{a}_{1}$ of different frac- (c) The error $b_{1}-\hat{b}_{1}$ of different fractional.

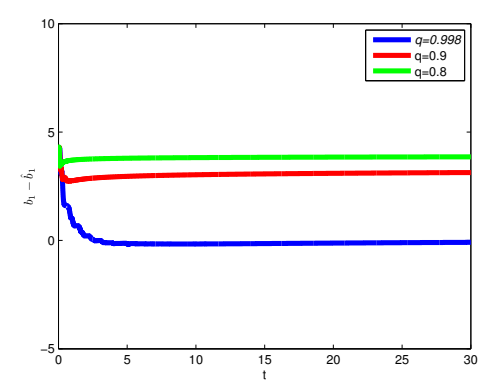

tional.

Figure 7: The related changes caused by different fractional order.

Remark 4.1. The fractional order $\alpha$ has a direct effect not only on the chaotic behavior of fractional-order nonlinear dynamical systems but also on the synchronization behavior of fractional-order systems. In $[4,30]$, the authors observed that the synchronization error decreases as the order $\alpha$ increases. However, the opposite phenomenon is also found in [38]. In this paper, we provide a more visible method to examine $\alpha$ 's impact on the fractional-order chaotic complex systems with unknown parameters. To this end, we consider three cases including $\alpha_{1}=0.998, \alpha_{2}=0.9$, and $\alpha_{3}=0.8$. All of the other settings remain unchanged as stated in the above numerical example. From Figure 7, we can conclude that the small value of the fractional order $\alpha$ would be harmful for identification of the unknown parameters although it has the beneficial effect on the synchronization state.

\section{Conclusions}

The objective of this paper is to investigate adaptive anti-synchronization of general fractional-order chaotic complex systems with all situations of unknown parameters. On the basis of the related results on the Caputo fractional derivative of quadratic functions and the adaptive control technique, the anti-synchronization controllers and the fractional-order adaptive laws of uncertain parameters are presented. It should be noted that quadratic Lyapunov functions play an important role in the analysis of the fractional-order error dynamical systems, which is different from those proposed in the existing works. Furthermore, the theoretical results are successfully applied to realize anti-synchronization between the fractional-order complex Lorenz and the fractional-order complex Lü systems with unknown parameters. The corresponding numerical simulations agree with the theoretical analysis. Therefore, it is believed that the proposed scheme will have bright prospect in practical applications. 


\section{Acknowledgment}

The authors are grateful to the editors and two anonymous referees for a very thorough reading of the manuscript and for kindly prompting improvements in presentation. This research is supported by NNSF of P. R. China (Grant Nos. 61503171, 61273088, 61603203, and 61403061), CPSF (Grant No. 2015M582091), NSF of Shandong Province (Grant Nos. ZR2016JL021 and ZR2016AB04), DSRF of Linyi University (Grant No. LYDX2015BS001), and the AMEP of Linyi University, P. R. China.

\section{References}

[1] S. K. Agrawal, S. Das, Projective synchronization between different fractional-order hyperchaotic systems with uncertain parameters using proposed modified adaptive projective synchronization technique, Math. Methods Appl. Sci., 37 (2014), 2164-2176. 1

[2] N. Aguila-Camacho, M. A. Duarte-Mermoud, J. A. Gallegos, Lyapunov functions for fractional order systems, Commun. Nonlinear Sci. Numer. Simul., 19 (2014), 2951-2957. 2.1, 2.2, 2.3

[3] D. Baleanu, G.-C. Wu, Y.-R. Bai, F.-L. Chen, Stability analysis of Caputo-like discrete fractional systems, Commun. Nonlinear Sci. Numer. Simul., 48 (2017), 520-530. 2.1

[4] S. Bhalekar, V. Daftardar-Gejji, Synchronization of different fractional order chaotic systems using active control, Commun. Nonlinear Sci. Numer. Simul., 15 (2010), 3536-3546. 4.1

[5] M. Caputo, Linear models of dissipation whose Q is almost frequency independent-II, Geophys. J. Int., 13 (1967), 529-539. 2.1

[6] L.-P. Chen, Y. Chai, R.-C. Wu, Lag projective synchronization in fractional-order chaotic (hyperchaotic) systems, Phys. Lett. A, 375 (2011), 2099-2110. 1

[7] J.-Y. Chen, C.-D. Li, T.-W. Huang, X.-J. Yang, Global stabilization of memristor-based fractional-order neural networks with delay via output-feedback control, Modern Phys. Lett. B, 2017 (2017), 19 pages. 2.1

[8] K. Diethelm, N. J. Ford, A. D. Freed, A predictor-corrector approch for the numerical solution of fractional differential equations, Nonlinear Dynam., 29 (2002), 3-22. 2.1

[9] A. M. A. El-Sayed, E. Ahmed, H. A. A. El-Saka, Dynamic properties of the fractional-order logistic equation of complex variables, Abstr. Appl. Anal., 2012 (2012), 12 pages. 1

[10] X. Gao, J.-B. Yu, Chaos in the fractional order periodically forced complex Duffing's oscillators, Chaos Solitons Fractals, 24 (2005), 1097-1104. 1

[11] A. K. Golmankhaneh, R. Arefi, D. Baleanu, Synchronization in a nonidentical fractional order of a proposed modified system, J. Vib. Control, 21 (2015), 1154-1161. 1

[12] A. Jajarmi, M. Hajipour, D. Baleanu, New aspects of the adaptive synchronization and hyperchaos suppression of a financial model, Chaos Solitons Fractals, 99 (2017), 285-296. 1

[13] C.-M. Jiang, S.-T. Liu, C. Luo, A new fractional-order chaotic complex system and its antisynchronization, Abstr. Appl. Anal., 2014 (2014), 12 pages. 1

[14] C.-M. Jiang, S.-T. Liu, D. Wang, Generalized combination complex synchronization for fractional-order chaotic complex systems, Entropy, 17 (2015), 5199-5217. 1

[15] A. Kiani-B, K. Fallahi, N. Pariz, H. Leung, A chaotic secure communication scheme using fractional chaotic systems based on an extended fractional Kalman filter, Commun. Nonlinear Sci. Numer. Simul., 14 (2009), 863-879. 1

[16] C.-G. Li, G.-R. Chen, Chaos and hyperchaos in the fractional-order Rössler equations, Phys. A, 341 (2004), 55-61. 1

[17] C.-G. Li, G.-R. Chen, Chaos in the fractional order Chen system and its control, Chaos Solitons Fractals, 22 (2004), 549-554. 1

[18] H.-L. Li, C. Hu, Y.-L. Jiang, Z.-L. Wang, Z.-D. Teng, Pinning adaptive and impulsive synchronization of fractional-order complex dynamical networks, Chaos Solitons Fractals, 92 (2016), 142-149. 1

[19] J. Liu, Complex modified hybrid projective synchronization of different dimensional fractional-order complex chaos and real hyper-chaos, Entropy, 16 (2014), 6195-6211. 1

[20] X.-J. Liu, L. Hong, L.-X. Yang, Fractional-order complex T system: bifurcations, chaos control, and synchronization, Nonlinear Dynam., 75 (2014), 589-602. 1

[21] J. Liu, S.-T. Liu, W. Li, Complex modified generalized projective synchronization of fractional-order complex chaos and real chaos, Adv. Difference Equ., 2015 (2015), 16 pages. 1

[22] S. Liu, X. Wu, X.-F. Zhou, W. Jiang, Asymptotical stability of Riemann-Liouville fractional nonlinear systems, Nonlinear Dynam., 86 (2016), 65-71. 2.1

[23] S. Liu, X.-F. Zhou, X.-Y. Li, W. Jiang, Asymptotical stability of Riemann-Liouville fractional singular systems with multiple time-varying delays, Appl. Math. Lett., 65 (2017), 32-39. 2.1

[24] J.-H. Luo, H. Liu, J.-F. Yang, Robust synchronization of uncertain fractional order chaotic systems, IEICE Trans. Fundamentals, E98-A (2015), 2109-2116. 2.4

[25] C. Luo, X.-Y. Wang, Chaos generated from the fractional-order complex Chen system and its application to digital secure communication, Int. J. Modern Phys. C, 2013 (2013), 23 pages. 1 
[26] C. Luo, X.-Y. Wang, Chaos in the fractional-order complex Lorenz system and its synchronization, Nonlinear Dynam., 71 (2013), 241-257. 1

[27] P. Muthukumar, P. Balasubramaniam, Feedback synchronization of the fractional order reverse butterfly-shaped chaotic system and its application to digital cryptography, Nonlinear Dynam., 74 (2013), 1169-1181. 1

[28] Z. M. Odibat, Adaptive feedback control and synchronization of non-identical chaotic fractional order systems, Nonlinear Dynam., 60 (2010), 479-487. 1

[29] H.-Y. Qin, C.-H. Zhang, T.-X. Li, Y. Chen, Controllability of abstract fractional differential evolution equations with nonlocal conditions, J. Math. Computer Sci., 17 (2017), 293-300. 2.1

[30] G.-Q. Si, Z.-Y. Sun, H.-Y. Zhang, Y.-B. Zhang, Parameter estimation and topology identification of uncertain fractional order complex networks, Commun. Nonlinear Sci. Numer. Simul., 17 (2012), 5158-5171. 4.1

[31] J.-W. Sun, W. Deng, G.-Z. Cui, Y.-F. Song, Real combination synchronization of three fractional-order complex-variable chaotic systems, Optik, 127 (2016), 11460-11468. 1

[32] H. Targhvafard, G. H. Enjace, Phase and anti-phase synchronization of fractional-order chaotic systems via active control, Commun. Nonlinear Sci. Numer. Simul., 16 (2011), 4079-4088. 1

[33] Z. Wang, X. Huang, N. Li, X.-N. Song, Image encryption based on a delayed fractional-order chaotic logistic system, Chin. Phys. B, 21 (2012), 6 pages. 1

[34] Z. Wang, X. Huang, G.-D. Shi, Analysis of nonlinear dynamics and chaos in a fractional order financial system with time delay, Comput. Math. Appl., 62 (2011), 1531-1539. 1

[35] G.-C. Wu, D. Baleanu, Chaos synchronization of the discrete fractional logistic map, Signal Process., 102 (2014), 96-99. 1

[36] G.-C. Wu, D. Baleanu, W.-H. Luo, Lyapunov functions for Riemann-Liouville-like fractional difference equations, Appl. Math. Comput., 314 (2017), 228-236. 2.1

[37] G.-C. Wu, D. Baleanu, H.-P. Xie, F.-L. Chen, Chaos synchronization of fractional chaotic maps based on the stability condition, Phys. A, 460 (2016), 374-383. 1

[38] X.-J. Wu, D.-R. Lai, H.-T. Lu, Generalized synchronization of the fractional-order chaos in weighted complex dynamical networks with nonidentical nodes, Nonlinear Dynam., 69 (2012), 667-683. 4.1

[39] H.-Q. Wu, X.-X. Zhang, S.-H. Xue, L.-F. Wang, Y. Wang, LMI conditions to global Mittag-Leffler stability of fractionalorder neural networks with impulses, Neurocomputing, 193 (2016), 148-154. 2.1

[40] L.-X. Yang, J. Jiang, Complex dynamical behavior and modified projective synchronization in fractional-order hyper-chaotic complex Lü system, Chaos Solitons Fractals, 78 (2015), 267-276. 1

[41] C. Yin, S. Dadras, S.-M. Zhong, Y.-Q. Chen, Control of a novel class of fractional-order chaotic systems via adaptive sliding mode control approach, Appl. Math. Model., 37 (2013), 2469-2483. 1

[42] Y.-P. Zhang, J.-T. Sun, Chaotic synchronization and anti-synchronization based on suitable separation, Phys. Lett. A, 330 (2004), 442-447. 1 